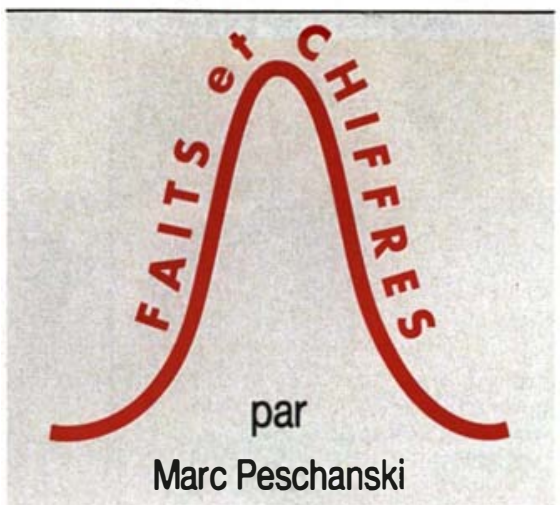

médecine/sciences $1995 ; 11: 277$

\title{
La Society for Neuroscience, comme un ouragan!
}

a Society for Neuroscience a tenu en novembre cette année, à Miami sous l'ouragan Gordon, ce qui, aujourd'hui de façon incontestable, est le grand rendez-vous mondial annuel des Neurosciences. Avec 22000 participants, soit une augmentation brutale de $50 \%$ en un an, le congrès de Washington en 1993 avait suscité les interrogations. Les neurosciences explosaient-elles ? La proximité des NIH expliquait-elle cette ruée soudaine ? Allions-nous dépasser bientôt les 25000 ? La Floride étant moins renommée pour ses centres de recherche biomédicale, le chiffre de participants de 1994 était très attendu, comme un indice du développement réel du domaine. $\mathrm{Ou}$ plutôt les chiffres, car le nombre de présents n'est pas forcément le plus significatif. A Washington, par exemple, il n'y avait que (!) un peu moins de 11000 présentations, soit une pour deux participants, alors que, les années précédentes, le chiffre approchait les 10000 pour 14000 à 15000 congressistes. Les "visiteurs" de Washington sem- blaient donc avoir fait une bonne partie de la différence.

Avec près de 10500 présentations annoncées, le congrès de Miami apparaissait, certes, inférieur à celui de l'année passée, mais déjà sensiblement supérieur aux précédents. Et avec 18000 participants, ce congrès a effectivement démontré que la pente ascendante rapide sensible avant 1993 $\left(\mathrm{m} / \mathrm{s} n^{\circ} 1\right.$, vol. $\left.8, p .71\right)$ se maintenait et, le chiffre est hautement significatif, près d'un tiers de ces participants - soit près de 6000 - étaient inscrits comme étudiants. Parmi ceux-ci, encore la moitié - soit près de 3000 étaient inscrits comme non-members et représentaient donc, en grand partie, les nouveaux arrivants du domaine. Ainsi, toutes les hypothèses formulées l'an dernier se sont révélées justes, ou fausses, en ceci que l'effet géographique a été vérifié, mais que l'explosion du domaine l'est aussi, rendant très réaliste la barre des 25000 : pour l'an 2000 peut-être, à moins que - les effets se conjuguant à nouveau - ce ne soit dès 1996 puisque le congrès retourne à Washington ! 